\title{
Agenesis of the corpus callosum
}

\section{with associated inter-hemispheric}

cyst and right frontal pachygyria

presenting with psychiatric symptoms in a Kenyan

\author{
HM Maru ${ }^{1}$, DIM Ndetei ${ }^{1}$, EO Amayo ${ }^{2}$, ZA Kaka ${ }^{3}$ \\ ${ }^{1}$ Department of Psychiatry, University of Nairobi, Kenya \\ ${ }^{2}$ Department of Medicine, University of Nairobi, Kenya \\ ${ }^{3}$ Department of Radiology, Nairobi Hospital, Kenya
}

\begin{abstract}
Summary
This case report presents a 26 year old man who had a history of childhood onset seizures, mild cognitive slowing and social withdrawal. He gradually developed symptoms of depression and attempted suicide once. He presented to the authors following a recurrence of his seizures. On examination they noted a normal general and neurological examination apart from some frontal lobe signs on mental status examination. He also had features of psychosis and labile mood. On CT brain scan he had agenesis of the corpus callosum (CC) with associated interhemispheric cyst and right frontal pachygyria. The authors suggest that clinicians in developing countries should be alert to organic disorders presenting with psychiatric symptoms.
\end{abstract}

Keywords: Electroencephalogram; Behavioural problems; Hallucinations; Developmental defect

Received: 25.07 .05

Accepted: 24.11 .05

A 26 year old African male was admitted with history of seeing spiders in his bedroom, allegations of verbal aggression and a recent relapse of seizures.

His birth was a full term, spontaneous non-eventful vertex delivery with immediately cry in a hospital with an Apgarscore of $9 / 10$, and reportedly normal milestones in the first three years, including walking at 9 months. At 3 years he had a first time afebrile tonic-clonic seizure, lost his speech, concentration appetite, and showed features suggestive of depression. At 7 years a psychological assessment showed performance on comprehension and general knowledge to be below average for his age. At 8 years, he regained speech with speech therapy; an electroencephalogram (EEG)

\section{Correspondence:}

Prof DM Ndetei, Department of Psychiatry, University of Nairobi,

P.O. Box 19676, Nairobi, Kenya

email: dmndetei@uonbi.ac.ke revealed epileptiform discharges, and he was subsequently put on sodium valproate with full remission. He was below average in class, dropping out at age 11 years. His parents then discontinued his anti-convulsants as he had been seizure free. He remained seizure free unit the age of 26 years when he consulted us following a recurrence. Further relevant psychosocial history included a suicide attempt by hanging at the age of 22 years, a history of unemployment and disinterest in all activities, but no clear history of major behavioural problems.

He was well kempt and the physical examination was normal, in particular he had no evidence of skull or spine deformities or cranial bruits. On neurological examination he was able to read fairly well, had no evidence of dysphasia, could draw a clock face, had no graphaesthesia and complete a line bisection task. He had no primitive reflexes (glabella tap, palmar mental, snout or grasp). Cranial nerve examination, fundoscopy, motor examination including tone, power and reflexes, sensory and cerebellar testing were all normal. His gait was normal including tandem gait and he was 


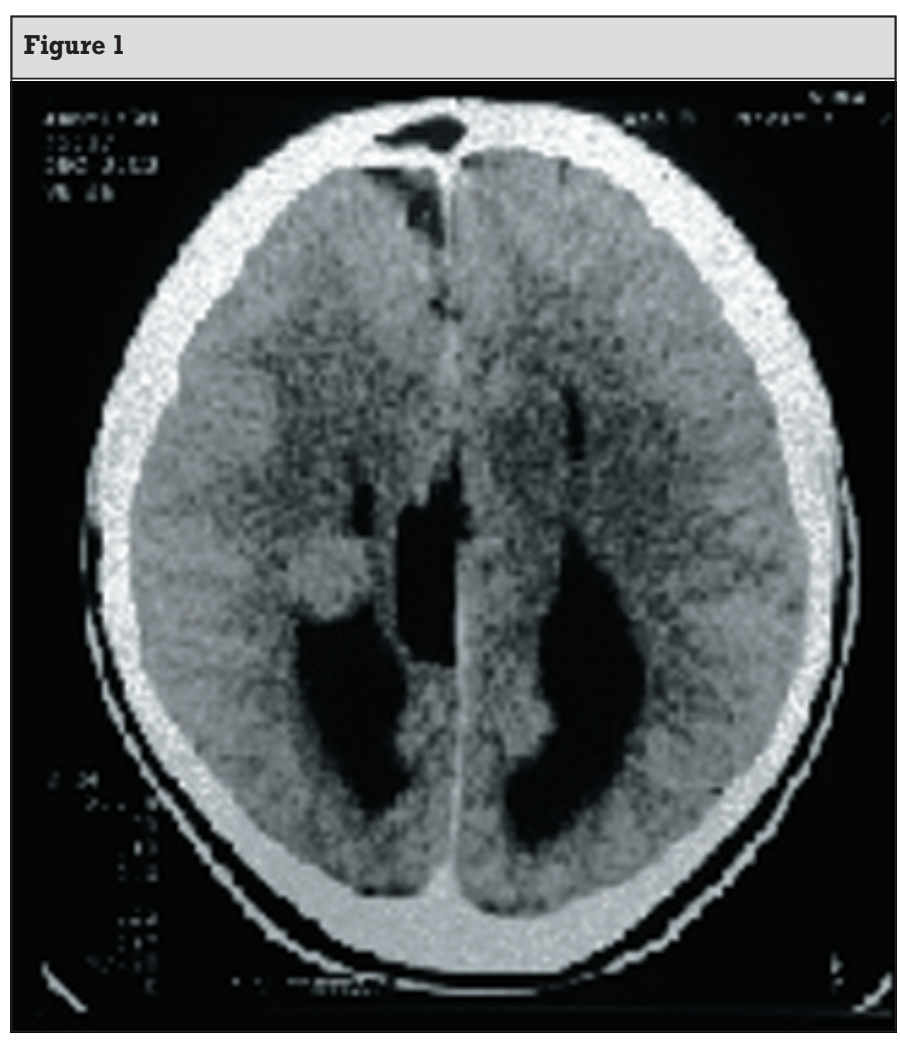

able to hop on one leg.

His mood was labile and he was argumentative, assertive and had flight of ideas and at same time expressed feelings of guilt and worthlessness. He complained of visual hallucinatins (more pronounced during the daytime) in the form of seeing spiders and mood incongruent auditory hallucinations. He was oriented except for time. Attention and concentration were poor and he had poor abstraction, immediate recall and only showed partial insight into his condition.

On investigation he had normal haematology, blood chemistry testing and EEG recordings were normal with no epileptiform, focal or generalized abnormalities.

CT Scan (Figures 1 and 2) of the head revealed agenesis of corpus callosum with associated interhemispheric cyst with right frontal pachygyria. No fat density could be identified in the mid-line using Hansfield Units (although a co-existing lipoma is often seen with this type of developmental defect).

His psychiatric symptoms did not respond to carbamazepine at a dose of $400 \mathrm{mg}$ a day. After two weeks he was put on haloperidol decanoate 50mg stat, with good response. He has maintained remission on a monthly dose of haloperidol decanoate 50mg.

This case manifested early in life with cerebral malfunction

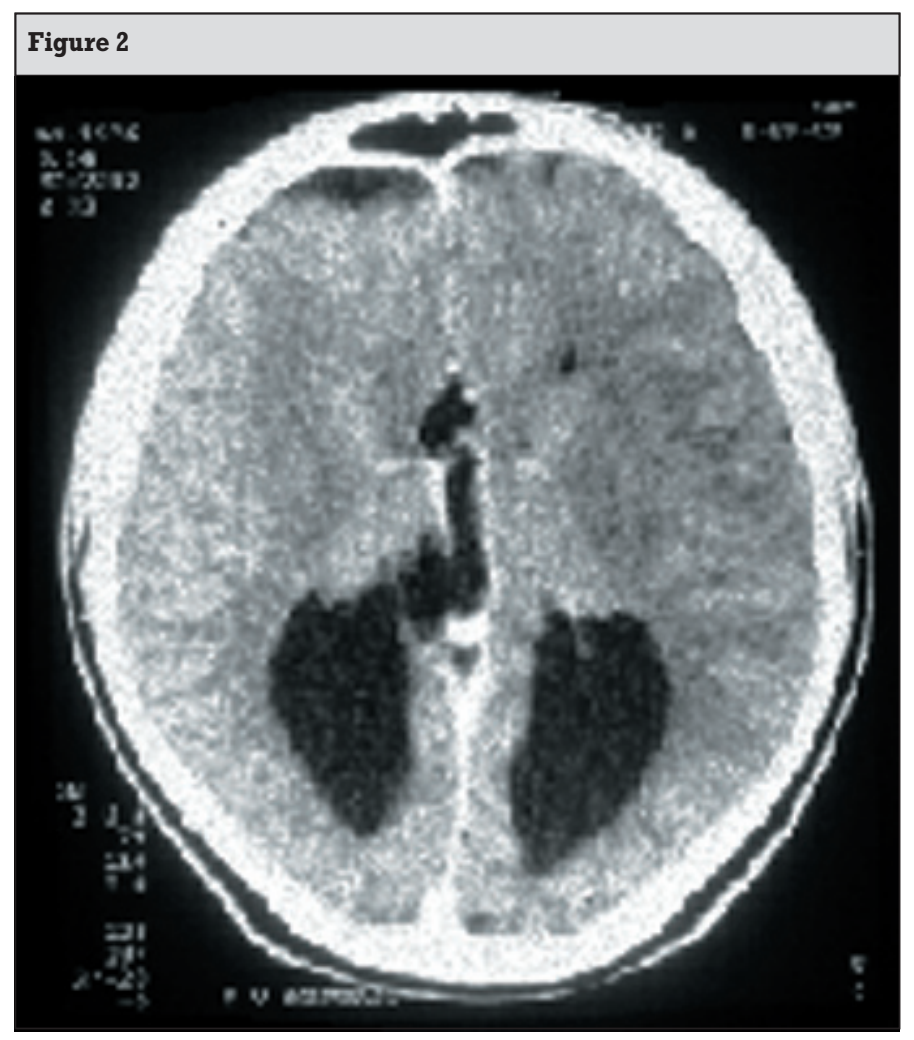

(seizures and mental retardation) and gradual development of psychiatric symptoms including symptoms of depression, mania and schizophrenia. The developmental defect was discovered at the age of 26 years. Corpus callosum developmental defects have been associated with major psychiatric disturbance, such as schizophrenia, depression, childhood behavioural disorders, bipolar disorders,

Asperger's syndrome, personality disorder and conversion symptoms. ${ }^{1}$ Suspected causes of corpus callosum agenesis include chromosomal defects, prenatal infections, toxins and metabolic disorders. ${ }^{1}$ Callosal agenesis has been associated with interhemispheric cysts and the association has a heterogeneous presentation. ${ }^{2}$ Clinicians in developing countries should be alert to organic disorders presenting with psychiatric symptoms.

\section{Reference}

1. David AS, Wacharasindhu A and Lishman WA. Severe Psychiatric disturbance and abnormalities of the courpus callosum; review and case series. Journal of Neurology, Neurosurgery and Psychiatry 1993; 56: 85-93.

2. Barkovich AJ, Simon EM, Walsh CA. Callosal agenesis with cyst. A better understanding and new classification. Neurology 2001; 56: 220- 227. 